International Journal of Pure and Applied Mathematics

Volume 99 No. 4 2015, 477-486

ISSN: 1311-8080 (printed version); ISSN: 1314-3395 (on-line version)

url: http://www.ijpam.eu

doi: http://dx.doi.org/10.12732/ijpam.v99i4.8

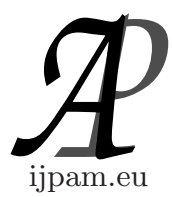

\title{
SOME FIXED POINT THEOREMS FOR MAPPINGS ATISFYING CONTRACTIVE CONDITIONS OF INTEGRAL TYPE IN MODIFIED INTUITIONISTIC UZZY METRIC SPACES
}

\author{
Saurabh Manro ${ }^{1}$, Shin Min Kang ${ }^{2} \S$ \\ ${ }^{1}$ School of Mathematics and Computer Applications \\ Thapar University \\ Patiala 147004, Punjab, INDIA \\ ${ }^{2}$ Department of Mathematics \\ Research Institute of Natural Science \\ Gyeongsang National University \\ Jinju, 660-701, KOREA
}

Abstract: In this paper, we prove some common fixed point theorems for occasionally weakly compatible mappings satisfying contractive conditions of integral type in modified intuitionistic fuzzy metric spaces.

AMS Subject Classification: 47H10, 54H25

Key Words: modified intuitionistic fuzzy metric space, occasionally weakly compatible mapping, common fixed point

\section{Introduction}

Atanassove [2] introduced and studied the concept of intuitionistic fuzzy sets as a generalization of fuzzy sets.

In 2004, Park [9] defined the notion of intuitionistic fuzzy metric spaces with the help of continuous $t$-norms and continuous $t$-conorms.

Received: December 26, 2014

(C) 2015 Academic Publications, Ltd.

${ }^{\S}$ Correspondence author 
Recently, in 2006, Alaca et al. [1] using the idea of intuitionistic fuzzy sets, defined the notion of intuitionistic fuzzy metric spaces with the help of continuous $t$-norm and continuous $t$-conorms as a generalization of fuzzy metric space due to Kramosil and Michálek [8]. In 2006, Türkoğlu et al. [13] proved Jungck's common fixed point theorem ([6]) in the setting of intuitionistic fuzzy metric spaces for commuting mappings. In 2006, Gregori et al. [5] showed that the topology induced by fuzzy metric coincides with topology induced by intuitionistic fuzzy metric.

In view of this observation, Saadati et al. [11], in 2008, reframed the idea of intuitionistic fuzzy metric spaces and proposed a new notion under the name of modified intuitionistic fuzzy metric spaces with the help of the notion of continuous $t$-representable.

In this paper, we prove some common fixed point theorems for occasionally weakly compatible mappings satisfying contractive conditions of integral type in modified intuitionistic fuzzy metric spaces.

\section{Preliminaries}

Definition 2.1. ([4]) A triangular norm (t-norm) on $L^{*}$ is a mapping $\Im:\left(L^{*}\right)^{2} \rightarrow L^{*}$ satisfying the following conditions: for all $x, x^{\prime}, y, y^{\prime}, z \in L^{*}$,

(i) $\Im\left(x, 1_{L^{*}}\right)=x$ (boundary condition);

(ii) $\Im(x, y)=\Im(y, x)$ (commutativity);

(iii) $\Im(x, \Im(y, z))=\Im(\Im(x, y), z)$ (associativity);

(iv) If $x \leq_{L^{*}} x^{\prime}$ and $y \leq_{L^{*}} y^{\prime}$, then $\Im(x, y) \leq_{L^{*}} \Im\left(x^{\prime}, y^{\prime}\right)$ (monotonicity), where $L^{*}=\left\{\left(x_{1}, x_{2}\right):\left(x_{1}, x_{2}\right) \in[0,1]^{2}\right.$ and $\left.x_{1}+x_{2} \leq 1\right\}$ and for every $\left(x_{1}, x_{2}\right)$ and $\left(y_{1}, y_{2}\right) \in L^{*},\left(x_{1}, x_{2}\right) \leq_{L^{*}}\left(y_{1}, y_{2}\right)$ if and only if $x_{1} \leq y_{1}$ and $x_{2} \geq y_{2}$.

We denote its units by $0_{L^{*}}=(0,1)$ and $1_{L^{*}}=(1,0)$.

Definition 2.2. ([4]) A continuous t-norm $\Im$ on $L^{*}$ is called continuous $t$-representable iff there exists a continuous $t$-norm $*$ and a continuous $t$-conorm $\diamond$ on $[0,1]$ such that for all $x=\left(x_{1}, x_{2}\right)$ and $y=\left(y_{1}, y_{2}\right) \in L^{*}, \Im(x, y)=$ $\left(x_{1} * y_{1}, x_{2} \diamond y_{2}\right)$.

Definition 2.3. ([11]) Let $M, N$ are fuzzy sets from $X^{2} \times(0, \infty)$ to $[0,1]$ such that $M(x, y, t)+N(x, y, t) \leq 1$ for all $x, y \in X$ and $t>0$. The 3-tuple $\left(X, \zeta_{M, N}, \Im\right)$ is said to be a modified intuitionistic fuzzy metric space if $X$ is an arbitrary non-empty set, $\Im$ is a continuous $t$-representable and $\zeta_{M, N}$ is a mapping from $X^{2} \times(0, \infty)$ to $L^{*}$ satisfying the following conditions: for all $x, y, z \in X$ and $t, s>0$,

(i) $\zeta_{M, N}(x, y, t)>_{L^{*}} 0_{L^{*}}$; 
(ii) $\zeta_{M, N}(x, y, t)=1_{L^{*}}$ if and only if $x=y$;

(iii) $\zeta_{M, N}(x, y, t)=\zeta_{M, N}(y, x, t)$;

(iv) $\zeta_{M, N}(x, y, t+s) \geq_{L^{*}} \Im\left(\zeta_{M, N}(x, z, t), \zeta_{M, N}(z, y, s)\right)$;

(v) $\zeta_{M, N}(x, y,):.(0, \infty) \rightarrow L^{*}$ is continuous.

In this case, $\zeta_{M, N}$ is called a modified intuitionistic fuzzy metric. Here, $\zeta_{M, N}(x, y, t)=(M(x, y, t), N(x, y, t))$ for all $x, y \in X$ and $t>0$.

Remark 2.4. ([10]) In a modified intuitionistic fuzzy metric space $\left(X, \zeta_{M, N}\right.$, $\Im)$, for any $t>0, \zeta_{M, N}(x, y, t)$ is non-decreasing with respect to $t$ in $\left(L^{*}, \leq_{L^{*}}\right)$ for all $x, y \in X$.

Definition 2.5. ([12]) Let $f$ and $g$ be self-mappings of a modified intuitionistic fuzzy metric space $\left(X, \zeta_{M, N}, \Im\right)$. Then the pair $(f, g)$ is said to be commuting if

$$
\zeta_{M, N}(f g x, g f x, t)=1_{L^{*}}
$$

for all $x \in X$ and $t>0$.

Definition 2.6. ([12]) Let $f$ and $g$ be self-mappings of a modified intuitionistic fuzzy metric space $\left(X, \zeta_{M, N}, \Im\right)$. Then the pair $(f, g)$ is said to be weakly commuting if

$$
\zeta_{M, N}(f g x, g f x, t) \geq_{L^{*}} \zeta_{M, N}(f x, g x, t)
$$

for all $x \in X$ and $t>0$.

Definition 2.7. ([11], [12]) Let $f$ and $g$ be self-mappings of a modified intuitionistic fuzzy metric space $\left(X, \zeta_{M, N}, \Im\right)$. Then the pair $(f, g)$ is said to be compatible if

$$
\lim _{n \rightarrow \infty} \zeta_{M, N}\left(f g x_{n}, g f x_{n}, t\right)=1_{L^{*}}
$$

for all $t>0$, whenever $\left\{x_{n}\right\}$ is a sequence in $X$ such that $\lim _{n \rightarrow \infty} f x_{n}=$ $\lim _{n \rightarrow \infty} g x_{n}=u$ for some $u \in X$.

Definition 2.8. ([11], [12]) Let $f$ and $g$ be self-mappings of a modified intuitionistic fuzzy metric space $\left(X, \zeta_{M, N}, \Im\right)$. Then the pair $(f, g)$ is is said to be weakly compatible if they commute at the coincidence points, that is, if $f u=g u$ for some $u \in X$, then $f g u=g f u$.

It is easy to see that compatible mappings are weakly compatible but converse is not true.

Definition 2.9. ([3], [7]) Let $f$ and $g$ be self-mappings of a modified intuitionistic fuzzy metric space $\left(X, \zeta_{M, N}, \Im\right)$. Then the pair $(f, g)$ is said to be 
occasionally weakly compatible if there exists a point $x \in X$ which is coincidence point of $f$ and $g$ at which $f$ and $g$ commute.

\section{Main Results}

Now, we prove common fixed point theorems for three mappings.

Theorem 3.1. Let $\left(X, \zeta_{M, N}, \Im\right)$ be a modified intuitionistic fuzzy metric space. Suppose that $f, g$ and $h$ are three self-mappings on $X$ satisfying the following conditions:

$$
\int_{0}^{\zeta_{M, N}(f x, g y, t)} \phi(t) d t \leq_{L^{*}} \int_{0}^{P 1} \phi(t) d t
$$

for all $x, y \in X$ and $\alpha, \beta, \gamma$ are non-negative reals numbers with $\alpha+2 \beta+2 \gamma<1$, where

$$
\begin{aligned}
P 1= & \alpha \zeta_{M, N}(h x, h y, t)+\beta\left[\zeta_{M, N}(f x, h x, t)+\zeta_{M, N}(g y, h y, t)\right] \\
& +\gamma\left[\zeta_{M, N}(h x, g y, t)+\zeta_{M, N}(h y, f x, t)\right]
\end{aligned}
$$

and $\phi: \mathbb{R}^{+} \rightarrow \mathbb{R}$ is a Lebesgue-integrable mapping which is summable, nonnegative and $\int_{0}^{\epsilon} \phi(t) d t>0$ for each $\epsilon>0$.

Assume that the pair $(f, h)$ or $(g, h)$ is occasionally weakly compatible.

Then $f, g$ and $h$ have a unique common fixed point.

Proof. Suppose that the pair $(f, h)$ is occasionally weakly compatible. Then there exists an element $u \in X$ such that $f u=h u$ and $f h u=h f u$.

Now, we prove that $f u=g u$. Indeed, by inequality $(C 1)$, we get

$$
\begin{aligned}
\int_{0}^{\zeta_{M, N}(f u, g u, t)} \phi(t) d t & \leq_{L^{*}} \int_{0}^{P 2} \phi(t) d t \\
& =\int_{0}^{\beta \zeta_{M, N}(g u, f u, t)+\gamma \zeta_{M, N}(f u, g u, t)} \phi(t) d t \\
& <L^{*} \int_{0}^{\zeta_{M, N}(f u, g u, t)} \phi(t) d t,
\end{aligned}
$$

where

$$
\begin{aligned}
P 2= & \alpha \zeta_{M, N}(h u, h u, t)+\beta\left[\zeta_{M, N}(f u, h u, t)+\zeta_{M, N}(g u, h u, t)\right] \\
& +\gamma\left[\zeta_{M, N}(h u, g u, t)+\zeta_{M, N}(h u, f u, t)\right]
\end{aligned}
$$


which is a contradiction and hence $g u=f u=h u$.

Again, suppose that $f f u \neq f u$. Then by $(C 1)$, we have

$$
\begin{aligned}
\int_{0}^{\zeta_{M, N}(f f u, g u, t)} \phi(t) d t & \leq_{L^{*}} \int_{0}^{P 3} \phi(t) d t \\
& =\int_{0}^{\alpha \zeta_{M, N}(f f u, g u, t)+2 \gamma \zeta_{M, N}(f f u, g u, t)} \phi(t) d t \\
& <_{L^{*}} \int_{0}^{\zeta_{M, N}(f f u, g u, t)} \phi(t) d t
\end{aligned}
$$

where

$$
\begin{aligned}
P 3= & \alpha \zeta_{M, N}(h f u, h u, t)+\beta\left[\zeta_{M, N}(f f u, h f u, t)+\zeta_{M, N}(g u, h u, t)\right] \\
& +\gamma\left[\zeta_{M, N}(h f u, g u, t)+\zeta_{M, N}(h u, f f u, t)\right]
\end{aligned}
$$

which is a contradiction and hence $f f u=f u=h f u$.

Now, suppose that $g f u \neq f u$. Then by $(C 1)$, we have

$$
\begin{aligned}
\int_{0}^{\zeta_{M, N}(f u, g f u, t)} \phi(t) d t & \leq L^{*} \int_{0}^{P 4} \phi(t) d t \\
& =\int_{0}^{\beta \zeta_{M, N}(g f u, f u, t)+\gamma \zeta_{M, N}(f u, g f u, t)} \phi(t) d t \\
& <L^{*} \int_{0}^{\zeta_{M, N}(f u, g f u, t)} \phi(t) d t,
\end{aligned}
$$

where

$$
\begin{aligned}
P 4= & \alpha \zeta_{M, N}(h u, h f u, t)+\beta\left[\zeta_{M, N}(f u, h u, t)+\zeta_{M, N}(g f u, h f u, t)\right] \\
& +\gamma\left[\zeta_{M, N}(h u, g f u, t)+\zeta_{M, N}(h f u, f u, t)\right]
\end{aligned}
$$

which is a contradiction and hence $g f u=f u$. Put $f u=g u=h u=z$. Therefore, $z$ is a common fixed point of $f, g$ and $h$.

Similarly, if the pair $(g, h)$ is occasionally weakly compatible, then $f, g$ and $h$ have a common fixed point.

Finally, let $z$ and $w(z \neq w)$ be two common fixed points of $f, g$ and $h$. 
Then from $(C 1)$, we have

$$
\begin{aligned}
\int_{0}^{\zeta_{M, N}(z, w, t)} \phi(t) d t & =\int_{0}^{\zeta_{M, N}(f z, g w, t)} \phi(t) d t \\
& \leq L^{*} \int_{0}^{P 5} \phi(t) d t \\
& =\int_{0}^{\alpha \zeta_{M, N}(z, w, t)+2 \gamma \zeta_{M, N}(z, w, t)} \phi(t) d t \\
& <L_{L^{*}} \int_{0}^{\zeta_{M, N}(z, w, t)} \phi(t) d t
\end{aligned}
$$

where

$$
\begin{aligned}
P 5= & \alpha \zeta_{M, N}(h z, h w, t)+\beta\left[\zeta_{M, N}(f z, h z, t)+\zeta_{M, N}(g w, h w, t)\right] \\
& +\gamma\left[\zeta_{M, N}(h z, g w, t)+\zeta_{M, N}(h w, f z, t)\right]
\end{aligned}
$$

which is a contradiction and hence $z=w$. Thus the common fixed point is unique. This completes the proof.

If we put $\phi(t)=1$ in Theorem 3.1, we get the following corollary:

Corollary 3.2. Let $\left(X, \zeta_{M, N}, \Im\right)$ be a modified intuitionistic fuzzy metric space. Suppose that $f, g$ and $h$ are three self-mappings on $X$ satisfying the following conditions:

$$
\begin{aligned}
\zeta_{M, N} & (f x, g y, t) \\
\leq_{L^{*}} & \alpha \zeta_{M, N}(h x, h y, t)+\beta\left[\zeta_{M, N}(f x, h x, t)+\zeta_{M, N}(g y, h y, t)\right] \\
& +\gamma\left[\zeta_{M, N}(h x, g y, t)+\zeta_{M, N}(h y, f x, t)\right]
\end{aligned}
$$

for all $x, y \in X$ and $\alpha, \beta, \gamma$ are non-negative reals numbers with $\alpha+2 \beta+2 \gamma<1$.

Assume that the pair $(f, h)$ or $(g, h)$ is occasionally weakly compatible.

Then $f, g$ and $h$ have a unique common fixed point.

Next, we prove common fixed point theorems for four mappings.

Theorem 3.3. Let $\left(X, \zeta_{M, N}, \Im\right)$ be a modified intuitionistic fuzzy metric space. Suppose that $f, g, h$ and $k$ are four self-mappings on $X$ satisfying the following conditions:

$$
\int_{0}^{\zeta_{M, N}(f x, g y, t)} \phi(t) d t \leq_{L^{*}} \int_{0}^{Q 1} \phi(t) d t
$$


for all $x, y \in X$ and $\alpha, \beta, \gamma$ are non-negative reals numbers with $\alpha+2 \beta+2 \gamma<1$, where

$$
\begin{aligned}
Q 1= & \alpha \zeta_{M, N}(h x, k y, t)+\beta\left[\zeta_{M, N}(f x, h x, t)+\zeta_{M, N}(g y, k y, t)\right] \\
& +\gamma\left[\zeta_{M, N}(h x, g y, t)+\zeta_{M, N}(k y, f x, t)\right]
\end{aligned}
$$

and $\phi: \mathbb{R}^{+} \rightarrow \mathbb{R}$ is a Lebesgue-integrable mapping which is summable, nonnegative and $\int_{0}^{\epsilon} \phi(t) d t>0$ for each $\epsilon>0$.

Assume that the pairs $(f, h)$ and $(g, k)$ are occasionally weakly compatible.

Then $f, g, h$ and $k$ have a unique common fixed point.

Proof. Since pairs of mappings $(f, h)$ and $(g, k)$ are occasionally weakly compatible. Then there exists two points $u, v \in X$ such that $f u=h u$ and $f h u=h f u, g v=k v$ and $g k v=k g v$.

Now, we prove that $f u=g v$. Indeed, by $(C 2)$, we get

$$
\begin{aligned}
\int_{0}^{\zeta_{M, N}(f u, g v, t)} \phi(t) d t & \leq L^{*} \int_{0}^{Q 2} \phi(t) d t \\
& =\int_{0}^{\alpha \zeta_{M, N}(f u, g v, t)+2 \gamma \zeta_{M, N}(f u, g v, t)} \phi(t) d t \\
& <_{L^{*}} \int_{0}^{\zeta_{M, N}(f u, g v, t)} \phi(t) d t,
\end{aligned}
$$

where

$$
\begin{aligned}
Q 2= & \alpha \zeta_{M, N}(h u, k v, t)+\beta\left[\zeta_{M, N}(f u, h u, t)+\zeta_{M, N}(g v, k v, t)\right] \\
& +\gamma\left[\zeta_{M, N}(h u, g v, t)+\zeta_{M, N}(k v, f u, t)\right],
\end{aligned}
$$

which is a contradiction and hence $g v=f u=h u=k v$.

Again, suppose that $f f u=f h u=h f u \neq f u$. Then by $(C 2)$, we have

$$
\begin{aligned}
\int_{0}^{\zeta_{M, N}(f f u, g v, t)} \phi(t) d t & \leq_{L^{*}} \int_{0}^{Q 3} \phi(t) d t \\
& =\int_{0}^{\alpha \zeta_{M, N}(f f u, g v, t)+2 \gamma \zeta_{M, N}(f f u, g v, t)} \phi(t) d t \\
& <_{L^{*}} \int_{0}^{\zeta_{M, N}(f f u, g v, t)} \phi(t) d t
\end{aligned}
$$

where

$$
\begin{aligned}
Q 3= & \alpha \zeta_{M, N}(h f u, k v, t)+\beta\left[\zeta_{M, N}(f f u, h f u, t)+\zeta_{M, N}(g v, k v, t)\right] \\
& +\gamma\left[\zeta_{M, N}(h f u, g v, t)+\zeta_{M, N}(k v, f f u, t)\right]
\end{aligned}
$$


which is a contradiction and hence $f f u=f u=h f u=f h u$.

Similarly, we obtain $g f u=k f u=f u$. Put $f u=z$. Therefore, $z$ is a common fixed point of $f, g, h$ and $k$.

Finally, let $z$ and $w(z \neq w)$ be two common fixed points of $f, g, h$ and $k$. Then from $(C 2)$, we have

$$
\begin{aligned}
\int_{0}^{\zeta_{M, N}(z, w, t)} \phi(t) d t & =\int_{0}^{\zeta_{M, N}(f z, g w, t)} \phi(t) d t \\
& \leq L^{*} \int_{0}^{Q 4} \phi(t) d t \\
& =\int_{0}^{\alpha \zeta_{M, N}(z, w, t)+2 \gamma \zeta_{M, N}(z, w, t)} \phi(t) d t \\
& <_{L^{*}} \int_{0}^{\zeta_{M, N}(z, w, t)} \phi(t) d t,
\end{aligned}
$$

where

$$
\begin{aligned}
Q 4= & \alpha \zeta_{M, N}(h z, k w, t)+\beta\left[\zeta_{M, N}(f z, h z, t)+\zeta_{M, N}(g w, k w, t)\right] \\
& +\gamma\left[\zeta_{M, N}(h z, g w, t)+\zeta_{M, N}(k w, f z, t)\right]
\end{aligned}
$$

which is a contradiction and hence $z=w$. Thus, the common fixed point is unique. This completes the proof.

If we put $\phi(t)=1$ in Theorem 3.3, we get the following corollary:

Corollary 3.4. Let $\left(X, \zeta_{M, N}, \Im\right)$ be a modified intuitionistic fuzzy metric space. Suppose that $f, g, h$ and $k$ are four self-mappings on $X$ satisfying the following conditions:

$$
\begin{aligned}
\zeta_{M, N} & (f x, g y, t) \\
\leq_{L^{*}} & \alpha \zeta_{M, N}(h x, k y, t)+\beta\left[\zeta_{M, N}(f x, h x, t)+\zeta_{M, N}(g y, k y, t)\right] \\
& +\gamma\left[\zeta_{M, N}(h x, g y, t)+\zeta_{M, N}(k y, f x, t)\right]
\end{aligned}
$$

for all $x, y \in X$ and $\alpha, \beta, \gamma$ are non-negative reals numbers with $\alpha+2 \beta+2 \gamma<1$.

Assume that the pairs $(f, h)$ and $(g, k)$ are occasionally weakly compatible.

Then $f, g, h$ and $k$ have a unique common fixed point.

Example 3.5. Let $X=[0, \infty)$ with the modified intuitionistic fuzzy metric

$$
\zeta_{M, N}(x, y, t)=\left(\frac{t}{t+|x-y|}, \frac{|x-y|}{t+|x-y|}\right)
$$


for all $t>0$ and $x, y \in X$. Define

$$
\begin{aligned}
& f x=g x= \begin{cases}0, & x \in[0,1), \\
1, & x \in[1, \infty),\end{cases} \\
& h x=\left\{\begin{array}{ll}
3, & x \in[0,1), \\
\frac{1}{x}, & x \in[1, \infty),
\end{array} \quad k x= \begin{cases}9, & x \in[0,1), \\
\frac{1}{\sqrt{x}}, & x \in[1, \infty) .\end{cases} \right.
\end{aligned}
$$

Clearly the pairs $(f, h)$ and $(g, k)$ are occasionally weakly compatible. Also if we define $\phi(x)=3 x^{2}$ and by taking $\alpha=\frac{1}{4}, \beta=\frac{1}{5}$ and $\gamma=\frac{1}{6}$, then all the hypotheses of Theorem 3.3 are satisfied and $x=1$ is a unique common fixed point of $f, g, h$ and $k$.

\section{References}

[1] C. Alaca, D. Türkoğlu, C. Yildiz, Fixed points in intuitionistic fuzzy metric spaces, Chaos Solitons Fractals, 29, No. 5 (2006), 1073-1078, doi: 10.1016/j.chaos.2005.08.066

[2] K.T. Atanassov, Intuitionistic fuzzy sets, Fuzzy Sets and Systems, 20, No. 1 (1986), 87-96, doi: 10.1016/S0165-0114(86)80034-3

[3] H. Chandra, A. Bhatt, Fixed point theorems for occasionally weakly compatible maps in probabilistic semi-metric space, Int. J. Math. Anal., 3, No. 12 (2009), 563-570.

[4] G. Deschrijver, C. Cornelis, E.E. Kerre, On the representation of intuitionistic fuzzy $t$-norm and $t$-conorms, IEEE Trans. Fuzzy System, 12, No. 1 (2004), 45-61, doi: 10.1109/TFUZZ.2003.822678

[5] V. Gregori, S. Romaguera, P. Veeramani, A note on intuitionistic fuzzy metric spaces, Chaos Solitons Fractals, 28, No. 4 (2006), 902-905, doi: 10.1016/j.chaos.2005.08.113

[6] G. Jungck, Commuting mappings and fixed points, Amer. Math. Monthly, 83, No. 4 (1976), 261-263, doi: 10.2307/2318216

[7] G. Jungck, B.E. Rhoades, Fixed point theorems for occasionally weakly compatible mappings, Fixed Point Theory, 7, No. 2 (2006), 286-296.

[8] I. Kramosil, J. Michálek, Fuzzy metric and statistical metric spaces, Kybernetika, 11, No. 5 (1975), 336-344. 
[9] J.H. Park, Intuitionistic fuzzy metric spaces, Chaos Solitons Fractals, 22, No. 5 (2004), 1039-1046, doi: 10.1016/j.chaos.2004.02.051

[10] R. Saadati, J.H. Park, On the intuitionistic topological spaces, Chaos Solitons Fractals, 27, No. 2 (2006), 331-344, doi: 10.1016/j.chaos.2005.03.019

[11] R. Saadati, S. Sedghi, N. Shobhe, Modified intuitionistic fuzzy metric spaces and some fixed point theorems, Chaos Solitons Fractals, 38, No. 1 (2008), 36-47, doi: 10.1016/j.chaos.2006.11.008

[12] M. Tanveer, M. Imdad, D. Gopal, D. Kumar, Common fixed point theorems in modified intuitionistic fuzzy metric spaces with common property (E.A), Fixed Point Theory Appl., 2012, No. 36 (2012), 12 pages, doi: 10.1186/1687-1812-2012-36

[13] D. Türkoğlu, C. Alaca, Y.J. Cho, C. Yildiz, Common fixed point theorems in intuitionistic fuzzy metric spaces, J. Appl. Math Comput., 22, No. 1-2 (2006), 411-424, doi: 10.1007/BF02896489. 